\title{
LA CARTOGRAFÍA HISTÓRICA Y EL ESTUDIO DE LOS PAISAJES CULTURALES MARÍTIMOS. APLICACIÓN METODOLÓGICA AL CASO DE LA BAHIA DE CADIZ.
}

\section{The historical cartography and the study of the maritime cultural landscapes. Methodological application to the case of the bay of Cadiz.}

Recibido: 19/11/2018

Revisado: 15/05/2019
Aceptado: 29/05/2019

Publicado: 05/07/2019

\section{RESUMEN:}

En el presente trabajo presentamos dos casos que ejemplifican a la perfección la utilidad de la aplicación del estudio de la cartografía histórica para el análisis de los paisajes culturales marítimos. Esto es posible gracias a la interrelación y estudios comparativos de los datos que aportan los documentos cartográficos a través de las herramientas que ofrecen los SIGs.

\section{ABSTRACT:}

In this survey we present two cases that perfectly exemplify the usefulness of the application of the study of historical cartography for the analysis of maritime cultural landscapes. This is possible thanks to the interrelation and comparative studies of the data provided by the cartographic documents through the Geografic Information Sistems

\section{Palabras claves}

Cartografía histórica; Georreferenciación; SIG; Bahía de Cádiz; Paisaje marítimo.

\section{KEY WORDS}

Historical mapping; Georeferencing; GIS; Bay of Cadiz; Maritime landscape.

sole.gomezmunoz@alum.uca.es 


\section{UTILIDAD DE LA CARTOGRAFÍA HISTÓRICA}

Desde que Christer Westerdahl (1980) definiera en la década de los 80 un nuevo concepto científico, "The Maritime Cultural Landscapes" (los paisajes culturales Marítimos), han proliferado numerosos proyectos de investigación (Ash, 2007; Freire, 2014; Duncan y Gibbs, 2015; Phillips, 2003; Calvo et al., 2011; Russo et al., 2014) cuyo objetivo ha sido, y es, estudiar los diferentes elementos que componen una sociedad marítima concreta. Este concepto unificó todo el registro arqueológico, de procedencia subacuática y terrestre, vinculado a una cultura marítima; pues, en ambos casos, constituyen una evidencia directa de la actividad de estas comunidades, la cual tiene su impronta en el espacio marítimo y litoral.

La perspectiva analítica y propuesta metodológica que sugiere esta corriente va mucho más allá de un estudio simplista centrado en una sociedad marítima a través de los vestigios materiales, pues pretende comprender la evolución en el uso que el ser humano hace del espacio marítimo bajo una perspectiva global (Westerdahl, 1992:5; Ash, 2007:3).

Para este cometido, incluye en el análisis elementos del patrimonio material e inmaterial, para el cual, es importante un enfoque marítimo. Por otro lado, el método de estudio que esta línea propone se caracteriza, grosso modo, por la posibilidad de estudiar el paisaje marítimo en base a tres perspectivas bien diferenciadas: en primer lugar, plantea un acercamiento al paisaje cultural marítimo desde una perspectiva puramente material a partir del registro arqueológico. Un segundo enfoque viene determinado por la propia cultura marítima (Westerdahl, 2011:744), conformada por todos aquellos elementos cognitivos fruto de la experiencia y del profundo conocimiento del medio, el cual trasciende a diferentes generaciones y civilizaciones. Y por último tenemos un enfoque, en el que se enmarca nuestro trabajo, focalizado en el estudio puramente físico. Es decir, estudia el contexto espacial en donde se desarrolla y condiciona la actividad de la comunidad marítima en cuestión. Si bien es cierto, que esta nueva línea, además, propone un acercamiento a estas realidades desde una posición puramente marítima y náutica, atendiendo también a las necesidades y condicionantes que influyen en estas comunidades. Esta posición fomenta, lo que denomina Hunter (1994) como: "la visión de la tierra desde el mar"; la cual se encontraba subordinada, totalmente, bajo una tradicional visión puramente terrestre de los distintos yacimientos arqueológicos.

El estudio en torno al paisaje marítimo, en cuanto al contexto físico y geográfico se refiere, puede ser abordado, a nuestro parecer, desde diversas disciplinas, todas ellas complementarias entre sí. Una de ellas es la fuente sobre la que se asienta nuestro análisis: la cartografía histórica. Ésta constituye una fuente de primer orden para acometer un trabajo de índole histórico-arqueológica sobre las sociedades marítimas, gracias a la riqueza de información, tanto escrita ${ }^{1}$ como gráfica. Ya que la cartografía refleja la realidad del paisaje en un momento cronológico concreto.

Gracias a la ingente volúmen de información que concentra este tipo de documentos, éstos adquieren múltiples aplicaciones para estudiar el paisaje cultural marítimo, especialmente el litoral. Los mapas y planos antiguos nos permiten identificar, entre otros datos, la evolución morfológica de la línea de costa, que ha estado condicionada en los últimos siglos por todo tipo de agentes naturales y antrópicos, permitiéndonos establecer análisis comparativos en clave diacrónica (Alonso et al, 2011). En base a ellos, también podremos reinterpretar y contextualizar diferentes yacimientos arqueológicos (Cerezo, 2013); así como ayudar en la planificación de las intervenciones arqueológicas o actividades geoarqueológicas (Cerezo, 2016), incluso localizar estructuras hoy desaparecidas. Además de tener ese carácter multifuncional, los documentos cartográficos también guardan una clara finalidad para preservar el patrimonio histórico-arqueológico terrestre y subacuático, favoreciendo la elección de las medidas pertinentes para su conservación

Estas aplicaciones son posibles gracias a la correcta integración y tratamiento de los datos que nos aportan los mapas antiguos, y su combinación y comparación con testimonios procedentes de otras fuentes como la literaria, la arqueológica o la fotográfica. Todo ello es posible gracias a las herramientas que nos ofrecen los Sistemas de Información Geográfica como es la Georreferenciación, en primer lugar, seguida de toda una serie de herramientas que facilitan la integración de los datos y los distintos análisis espaciales, como pueden ser los

1 Es importante recordar, que la documentación escrita que suelen acompañar a los planos y mapas es fundamental para poder comprender e interpretar el documento, pues de esta forma permite contextualizarlo en base a la finalidad por la que fue producido. 
estudios de visibilidad, conectividad o accesibilidad a un puerto por ejemplo.

En el presente trabajo ${ }^{2}$ estudiamos la transformación de diferentes franjas costeras de la Bahía de Cádiz a partir de la cartografía histórica. Para ello hemos aplicado un método de estudio comparativo fundamentado en el tratamiento de los datos cartográficos a partir de las herramientas que ofrecen los Sistemas de Información Geográfica; softwares cada vez más en boga en los análisis espaciales de paisajes antiguos.

\section{GEORREFERENCIACIÓN DE CARTOGRAFÍA ANTIGUA}

El primer paso, para estudiar la evolución del litoral a través de los mapas antiguos, es seleccionar el material con el que se va a trabajar, atendiendo a ciertas características, pues no todos los documentos son susceptibles de ser georreferenciados y por tanto, analizados mediante las herramientas que ofrecen los SIG. Para llevar a cabo una correcta georreferenciación, los mapas y planos deben haber sido confeccionados utilizando una serie de técnicas e instrumental mucho más preciso y moderno, propio de finales del s. XVIII, fruto de la revolución científica. Podríamos decir, que a partir de estas fechas, los mapas y planos adquieren mayor coherencia con la realidad. Sin embargo, ante tal requisito, no pretendemos desestimar el valor documental de los mapas de fechas anteriores, pues, en muchas ocasiones, resultan de utilidad al ser producciones previas a las grandes transformaciones del espacio. De este modo nos permite documentar determinadas estructuras que, posiblemente para el s. XVIII y XIX, ya habían desaparecido ${ }^{3}$

La georreferenciación de un mapa histórico a través de SIG, constituye una técnica que hoy en día supone una verdadera línea de estudio dentro del campo de la cartografía (Witmer et al., 2006;

2 Este artículo se enmarca dentro de nuestra tesis doctoral titulada Referentes para la navegación en la costa gaditana en época romana. Un acercamiento interdisciplinar. El caso de la bahía de Cádiz, actualmente en desarrollo dentro del programa de doctorado de Historia y Arqueología marítimas de EIDEMAR. Universidad de Cádiz.

3 Como pueden ser el trazado de antiguas murallas, restos arqueológicos que aún eran visibles en el s. XVI, o construcciones del medievo hoy inexistentes cuyo recuerdo únicamente nos llega a través de las fuentes literarias o, en este caso, cartográficas.
Cascón et al., 2018:204). Se trata de un método de posicionamiento espacial basado en asignar al plano antiguo un sistema de coordenadas conocido. Para ello, es necesario la elección de determinados puntos de apoyo en el documento antiguo, previamente escaneado a muy buena resolución, e identificar su homólogo en el plano actual (mediante el establecimiento de vínculos), cuyas coordenadas sí conocemos (Dávila y Camacho, 2012; Andrés, 2016; Cascón et al., 2018:204). Durante este proceso, el mapa a georreferenciar se irá solapando, y en cierto modo "desfigurando" para adaptarse al plano actual (Fig. 1), gracias a estos puntos de apoyo, que normalmente son elementos constructivos que apenas han sufrido modificaciones a lo largo del tiempo, y que hoy en día conservan la misma morfología.

Para el caso de la Bahía de Cádiz utilizamos elementos que aún mantienen su estructura como pueden ser: los baluartes de las murallas defensivas, fortificaciones costeras como el Castillo de San Lorenzo del Puntal (Puntales), el Fuerte de San Luis, el de San Sebastián o el de Santa Catalina del Puerto. También podemos utilizar iglesias como la de San José en Extramuros o el Monasterio de la Victoria en el Puerto de Santa María. De igual forma, los castillos también resultan útiles, como el Castillo de Luna en Rota, el de San Romualdo en San Fernando o el localizado en el islote de Sancti Petri. Como vemos, son edificaciones que se erigieron durante época Moderna y todavía hoy se encuentran presentes en el paisaje.

Un requisito indispensable para la elección de estos puntos es su dispersión en el espacio geográfico que vamos a tratar y la cantidad, pues, de dichos parámetros dependerá que la georreferenciación sea precisa en mayor o menor grado. Siguiendo estas recomendaciones, el error cuadramétrico ${ }^{4}$ total de la georreferenciación será el menor posible, por ende, el grado de coherencia de la transformación entre los distintos puntos de control será mayor (Cerezo, 2013). Tras haber generado los suficientes vínculos, el mapa histórico se transforma adquiriendo las coordenadas actuales.

Gracias a esta técnica, como ya citamos, es posible abordar toda una serie de análisis de tipo

$4 \mathrm{El}$ error cuadramético es la media de todos los errores cuadráticos medios (RMS) de cada punto. El error cuadrático es la diferencia entre el punto de partida, frente a la ubicación del punto de destino, un punto que se toma en el mapa a tratar con la posición de su homólogo en la cartografía actual. 


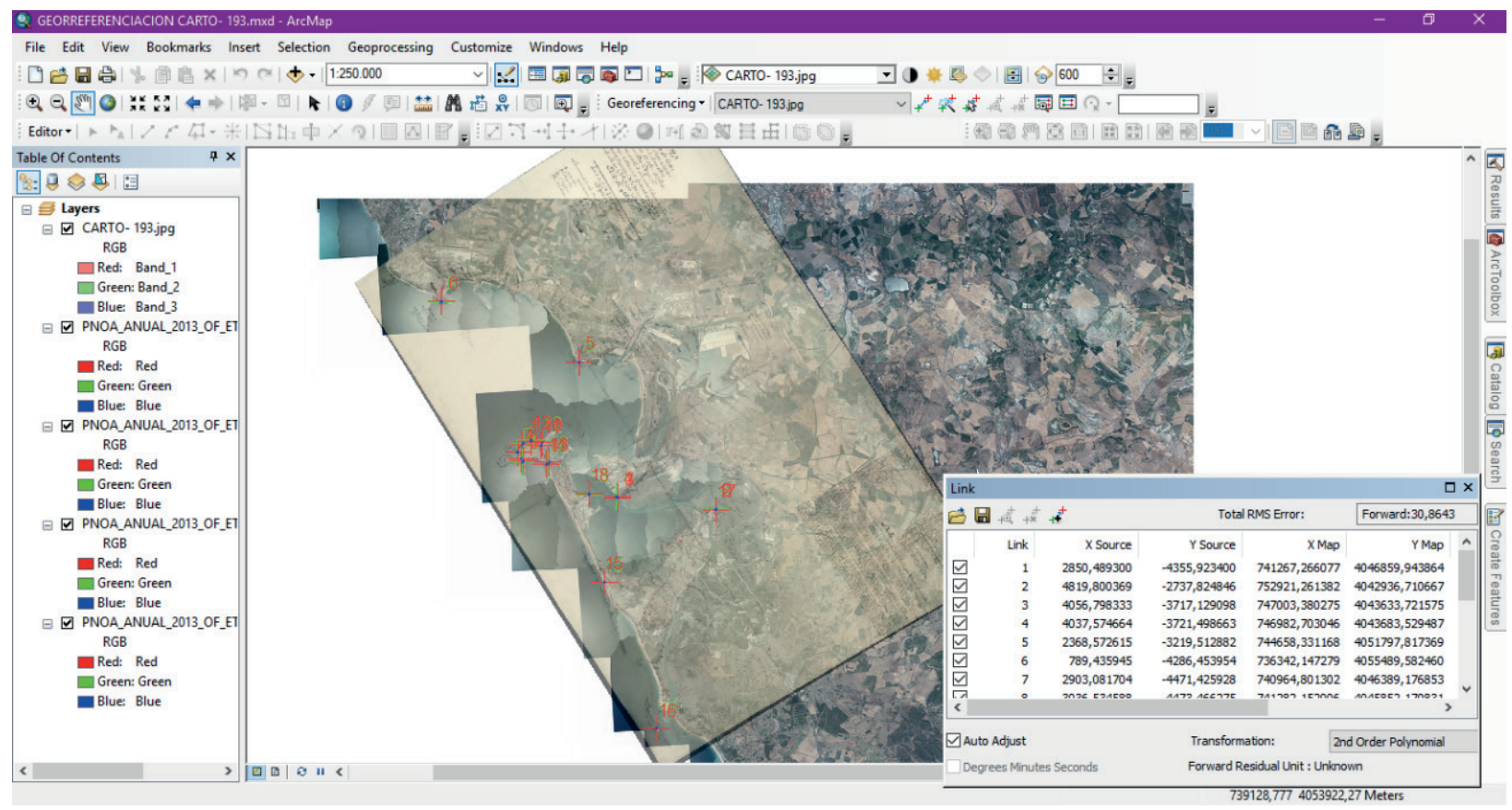

Figura 1.- Captura de pantalla que representa la aplicación de la herramienta Georreferencing de la aplicación Arcmap del software ArcGis. En concreto trabajamos con la versión 10.5. En la imagen se refleja la georreferenciación de un mapa del s. XIX con puntos de apoyo diseminados por el documento.

espacial, en base a múltiples elementos que son susceptibles de ser estudiados. Para nuestro trabajo, gracias a las herramientas de vectorización, hemos podido digitalizar distintos componentes que conforman el litoral de la bahía gaditana contextualizados en diferentes períodos, como: la línea de costa en pleamar y en bajamar, además de las planimetrías de obras portuarias y fortificaciones costeras. Gracias a estos datos podemos comprender el proceso evolutivo del paisaje costero, estableciendo análisis comparativos de diferentes áreas y en diferentes momentos cronológicos. Estos datos, a su vez, permiten interrelacionarlos con la geolocalización de yacimientos arqueológicos para su contextualización.

\section{ESTUDIO DE LOS DISTINTOS CASOS DE LA BAHÍA DE CÁDIZ.}

En los últimos siglos, la Bahía de Cádiz ha evolucionado ostensiblemente, atestiguándose dicha transformación en distintas fuentes. Esta gran rada experimenta profundos cambios a partir de la época Moderna, proceso que se agudiza a mediados del s. XIX y XX por la mecanización del transporte, la superpoblación de sus costas y el desarrollo de la industria naval (Cobos, 1993).
Esta profunda antropización, se materializó, no sólo en el crecimiento poblacional de las urbes próximas al litoral de la bahía, sino también en construcciones en plena línea de costa que en la mayoría de los casos supuso un mayor aterramiento de zonas en donde antaño estaban ocupadas por el mar. Como es el caso de la construcción de diques, muelles, embarcaderos, e infraestructura portuaria en general, para acondicionar los puertos ante la intensificación del comercio marítimo en el s. XVIII ${ }^{5}$ (Iglesias, 2007). Por otro lado, también tenemos las fortificaciones costeras y murallas para la defensa y protección de la Bahía ante ataques enemigos donde, en la mayoría de los casos, modifican los perfiles costeros ocultando los acantilados naturales y ganando terreno al mar. Asimismo, el crecimiento en la construcción naval incluye modificaciones en el paisaje ante la necesidad de habilitar espacios para construir careneros y astilleros. El crecimiento de la industria salinera también supuso un proceso clave que modifica el paisaje marítimo de la bahía. Este proceso ha provocado una intensa, y a la vez progresiva, desecación de las marismas.

5 Recordemos la intensificación del comercio marítimo al que asiste Cádiz durante esta centuria con el traslado de la Casa de la Contratación, en donde la ciudad pasa a controlar el monopolio del comercio americano, el principal flujo económico de la corona (Iglesias, 2017). 
Igualmente, los condicionantes naturales también han marcado considerablemente el paisaje, en ocasiones favoreciendo o condicionando las grandes obras antrópicas. Hablamos de la propia dinámica natural, la cual se reduce a dos grandes procesos claramente localizados, y que configuran y determinan la evolución natural de la costa. Nos referimos a la acusada colmatación provocada por el aporte de aluviones de los cursos fluviales que desembocan dentro de la Bahía, y la erosión que tiene lugar en toda la costa exterior que descarna acantilados y playas (Martín et al., 2003). A estos procesos se suman los mecanismos climáticos -temporales- y sísmicos -tsunamis- que afectan considerablemente al litoral.

Desde el s. XVI hasta la actualidad, hemos podido documentar, a través de la cartografía histórica, distintas zonas de la Bahía que han estado expuestas a mayores alteraciones. Entre ellas podemos destacar: la franja costera interna desde el puerto de Cádiz hasta la Ensenada de Puntales, y la desembocadura del Guadalete o la barra litoral de Valdelagrana. En las siguientes líneas abordaremos la problemática y cambios sufridos en ambos sectores, pues, consideramos que ambos casos ejemplifican a la perfección la utilidad de los documentos cartográficos para el estudio de paisajes antiguos; tal y como afirmaba Philippe Blanchemanche (2000): "los mapas históricos se consideran como testimonios de un espacio en un momento intermedio entre la antigüedad y la actualidad”.

\section{Las Desembocaduras de los ríos Guadalete y} San Pedro y la flecha de Valdelagrana.

Esta es una de las zonas de la Bahía que más modificaciones ha experimentado en el devenir de los siglos. En dicha área, localizada en la bahía exterior, se ha podido determinar su naturaleza geomorfológica durante la Antigüedad gracias a los recientes estudios históricos y geoarqueológicos. (Alonso et al., 2014; López et al., 2013), los cuales han favorecido el estudio paleotopográfico del antiguo estuario del Guadalete ${ }^{6}$. En base a la documentación, este curso desaguaba en un amplio lago

6 Trabajos recientes sostienen que la actual desembocadura del Guadalete fue una obra de ingeniería romana impulsada por Lucio Cornelio Balbo, el Menor, en el año 19 a. C.; de esta manera, los autores proponen el estuario del río San Pedro como la desembocadura originaria del Guadalete. que, para tiempos históricos, ya se encontraría cercado por una flecha litoral -la de Valdelagrana-, fruto del depósito de material en suspensión arrastrado por la propia deriva oceánica. Esta barra favorecería la colmatación de este estuario, cerrándolo poco a poco; impidiendo, de este modo, la salida de sedimentos arrastrados por la corriente fluvial al mar. Es por ello que se concentran en la desembocadura y provocan la progresiva progradación de la zona. Esta flecha es la que actualmente articula y configura la desembocadura del rio Guadalete y San Pedro; y entre ambos, fruto de la colmatación, se va generando una gran extensión de marismas, idóneas para la explotación salinera.

Este proceso de colmatación es posible visualizarlo en distintos documentos antiguos. Aparentemente, en el s. XVIII el antiguo estuario del Guadalete parecía estar en proceso de sedimentación, documentándose pequeñas láminas de agua en el interior. Un documento cartográfico en el que podemos apreciar no solo la naturaleza geomorofológica del estuario, sino también de toda la Bahía en general es la carta náutica del Ingeniero Don José Barnola fechada en 1743, de la que se dispone una copia fechada unos años más tarde. En esta carta es posible reconocer fácilmente, por medio de una combinación de diferentes colores, las áreas con cotas más bajas que corresponden a los terrenos inundables, producto de la progradación, que parecen fusionar las zonas más altas. Este mapa, fácilmente nos recuerda la fisonomía de la antigua Bahía donde su característica insularidad, según nos detallaban las fuentes escritas, resultaba en el s. XVIII más que evidente y fácilmente perceptible (Fig. 2)

En lo referente a la flecha litoral de Valdelagrana, al igual que el estuario, se aprecia fácilmente su evolución. Esta barra litoral, en su origen, es producto de la deposición de material arrastrado por la dinámica litoral predominante en la costa atlántica de Andalucía, de clara direccionalidad norte-sur (Arruda, 2005). Sin embargo, la flecha que actualmente apreciamos, es el resultado de una superposición de barras, puesto que, debido a diversos episodios sísmicos catastróficos, ésta se fragmentaba redireccionando, a su vez, la desembocadura del rio (Alonso et al. ,2015). La que hoy en día observamos es, lógicamente, la última barra superpuesta tras la ruptura por un tsunami ocurrido en el año 881 d.C. (Alonso et al., 2014). Ésta, en los últimos siglos, ha ido experimentando determinados cambios, ya que ha ido recibiendo, progresivamente, más depósitos. Este proceso ha favorecido su proyección, 


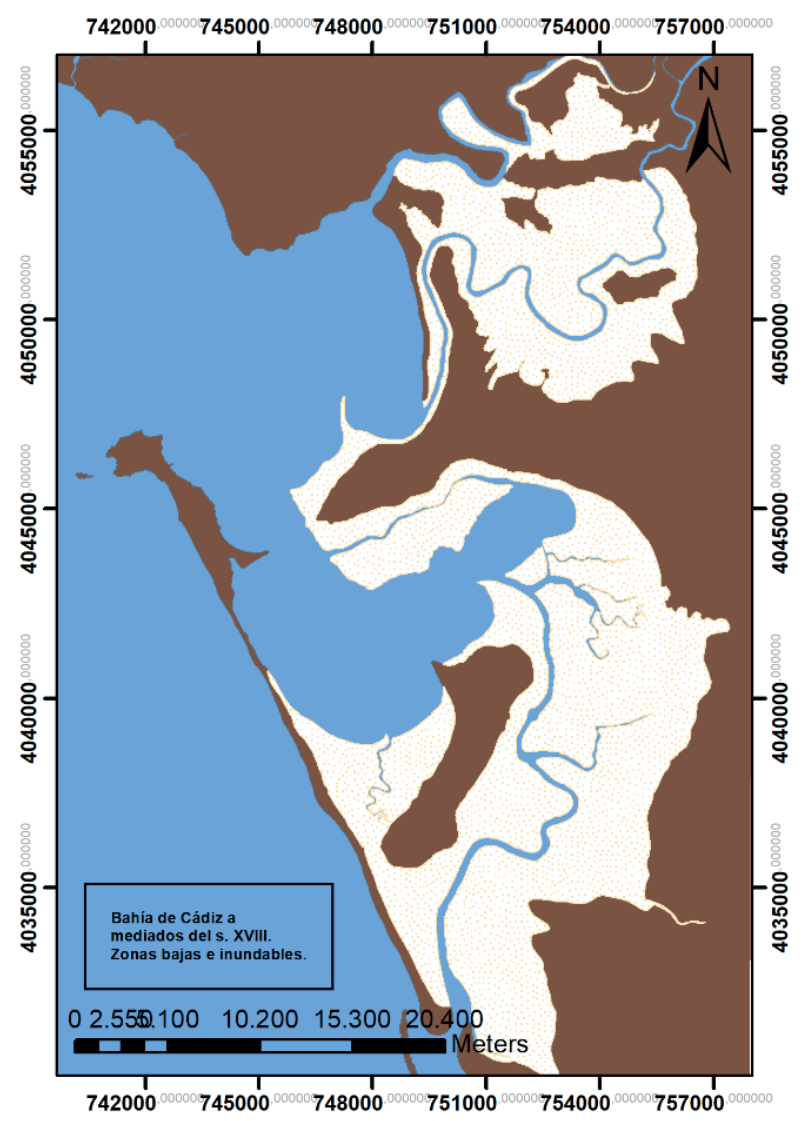

Figura 2.- Mapa original de mediados del s. XVIII, copia de la obra e Don Joseph Barnola (Centro Geográfico del Ejército. Ar. G- T8- C.4- 696) y a su lado el mismo ejemplar georreferenciado, del cual hemos procedido a digitalizar de esa época, además de distinguir las áreas en donde predominan las marismas producto de la progradación y acumulación de material arrastrado por los distintos cauces. Mapa de elaboración propia.

sobre todo hacia el oeste y sureste, ganando anchura y longitud. Un ejemplo que evidencia el carácter fluctuante de esta formación lo observamos en el mapa de la Bahía realizado por Don José Hidalgo a comienzos del s. XIX (Fig. 3).

En este ejemplar se registran las diferentes líneas de costa en pleamar y bajamar que el autor documenta en el año 1803, fecha en la que se confecciona el mapa. Esta línea a su vez es comparada con la que documentaron en 1789, fecha en la que el Brigadier don Vicente Tofiño realiza su obra, y en 1796. De este modo, podemos apreciar claramente la expansión de la barra, así como el desarrollo de la punta de Matagorda, por medio de la acumulación progresiva de sedimentos, y la propia desembocadura del Guadalete, progradándose también su barra. En resumen, apreciando esta imagen, podemos ver la rápida modificación de la flecha en menos de 15 años. Posiblemente esta transformación, en lo que se refiere al desarrollo de la barra y desembocadura del Guadalete, se pueda atribuir a las obras de reconducción del cauce del Guadalete tras haber dirigido anteriormente el caudal y unirlo con el del río San Pedro (Iglesias, 2017). Un ejemplo de cómo las obras hidráulicas pueden afectar a la morfología del litoral y quedar atestiguado en la cartografía histórica. Además de atestiguar los cambios fisionómicos del litoral, podemos percibir la conciencia, de los ingenieros y cartógrafos de esos años, de la cartografía como documento en el que representar el paisaje y poder comparar con situaciones de períodos anteriores.

\section{El caso del bajo de los Corrales y la ensenada de Puntales}

Esta zona constituye otro ejemplo cuya configuración morfológica se ha visto totalmente alterada. En este caso, la profunda incidencia antrópica actúa como factor determinante, sobre la dinámica natural, materializándose en la ejecución de aterramientos artificiales paralelos a toda la franja costera. Atendiendo a los cambios producidos, este proceso de aterramiento se puede dividir en 4 etapas:

$\left.1^{\mathrm{o}}\right)$ Primera regularización del perfil costero, camuflando, de este modo, los antiguos acantilados, con la construcción de las Puertas de Tierra y las murallas de Bahía Blanca en torno a los siglos XVI y XVII, durante el proceso de fortificación de Extramuros (Mapa base)

$\left.2^{\circ}\right)$ Obras de ampliación del puerto de Cádiz en el s. XVIII e implantación del ferrocarril en 1860 , que provocó el relleno de toda la zona que colinda con el puerto para la construcción de la estación ferroviaria (Color rojo).

$3^{\mathrm{o}}$ ) Ampliación, a comienzos del s. XX, de la infraestructura portuaria y áreas destinadas a Astilleros. Ello conllevó un mayor aterramiento alcanzando la Punta de las Vacas (Color amarillo).

$4^{\text {a }}$ ) Última etapa que corresponde con la situación actual. En el perfil costero podemos ver el cambio tan drástico que ha experimentado este sector litoral, en el cual se aprecia el relleno total de todo el bajo, regularizando la línea de costa hasta alcanzar el Castillo de Puntales, en donde actualmente se encuentra la Barriada de la Paz. De este modo se han perdido los antiguos acantilados, los bajos rocosos que colindaban con la línea de costa y la playa próxima al Castillo (Color negro). 


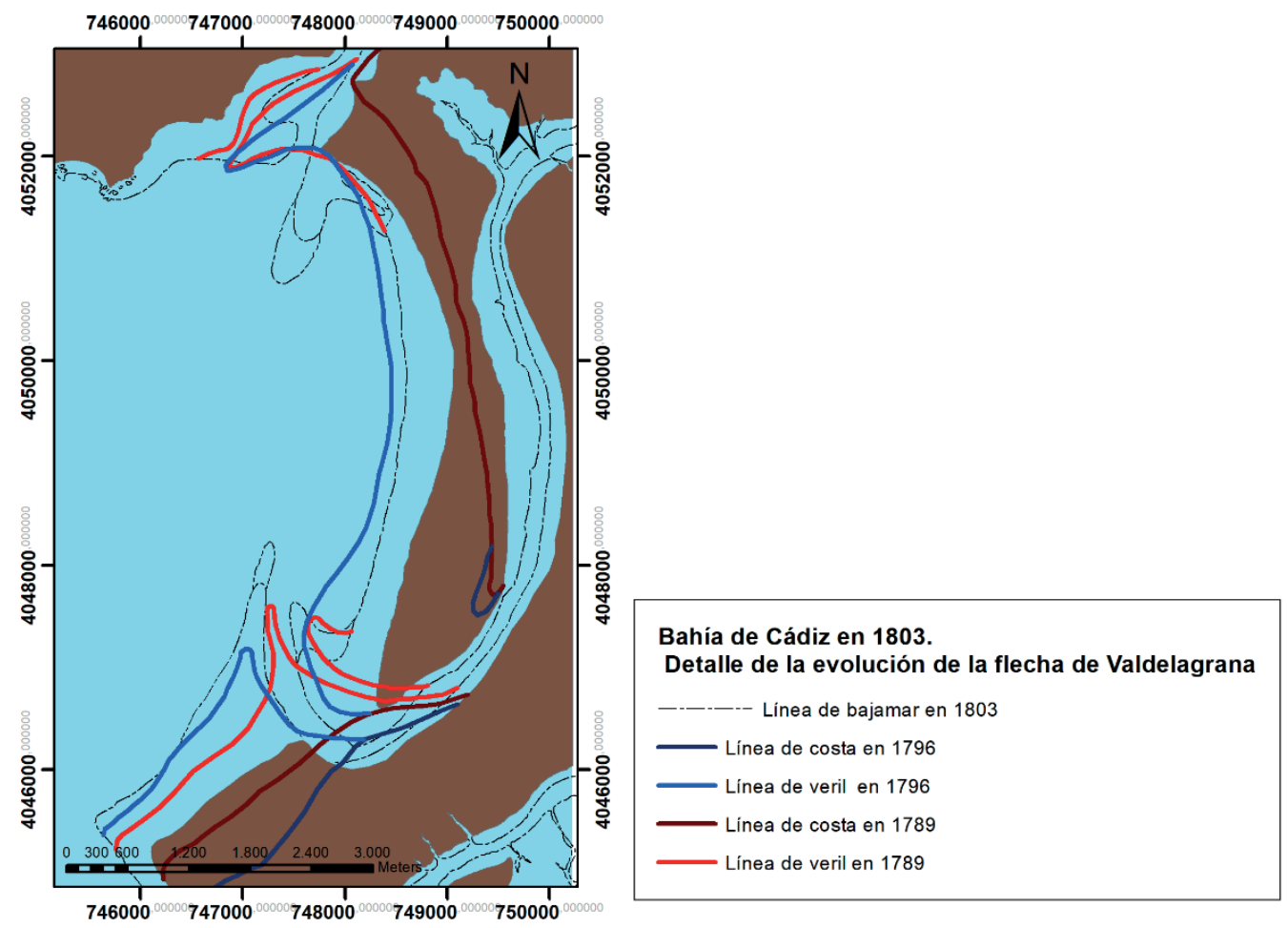

Figura 3.- Mapa general de la bahía original de José Hidalgo (Institut Cartogràfic i Geològic de Catalunya RM. 248375 ) realizado en los primeros años del s. XIX, junto al mapa confeccionado por medio del SIG en donde es posible analizar la evolución de la barra de Valdelagrana en diferentes momentos históricos. Mapa de elaboración propia.

Este fenómeno de aterramiento documentado en diferentes zonas del litoral también se contempla al sur de Puntales. En el s. XVIII, y bien entrado el s. XIX, se podía apreciar en el flanco meridional de esta punta de tierra, una suerte de pequeños arroyos o riachuelo que desembocaban en la ensenada homónima, conformando así una pequeña caletilla. Ésta, posiblemente, en la Antigüedad fuese más abrigada presentando la línea de costa mayor concavidad.

La cartografía es testigo de cómo este sector ha ido colmatándose y regulándose hasta nuestros días. En numerosas ocasiones, eran representadas pequeñas láminas de agua estancadas que ponían de manifiesto la presencia de una costa baja y pantanosa, la cual, que fue aprovechada durante el s. XIX para la construcción de diques ${ }^{7}$. Sin embargo, a partir de la segunda mitad del s. XX, en pleno auge constructivo, esta zona experimenta un cambio sustancial, dejando atrás la imagen de esa pequeña caleta de poco calado para pasar a una extensa superficie, fruto de los rellenos artificiales, en donde

7 A.G.S. (M.P.D, 57, 037), I.G.N. (S1-35-N-34) posteriormente se edificó el actual Barrio de la Laguna ${ }^{8}$ y el polígono industrial de la Zona Franca, más hacia el sur (Fig. 4).

Gracias a la superposición de las diferentes líneas de costa en orden cronológico, que podemos apreciar en la Figura 4, hemos podido advertir, en las dos zonas, los diferentes estadios evolutivos que ha experimentado hasta llegar a la situación actual. Esta progradación artificial, se ve favorecida por la propia dinámica natural que predomina en este sector de la Bahía, pues recordemos, que en esta franja litoral el azote de los agentes oceánicos no resulta tan agresivo como en la banda exterior; a lo que se une la propia progradación natural por los aportes fluviales.

Aprovechando el estudio de la evolución litoral de este sector, hemos llevado a cabo un ejercicio de contextualización de yacimientos arqueológicos de la zona. Para ello, hemos tomado como ejemplo yacimientos tan vinculados con las actividades marítimas y tan significativos en la arqueología gaditana como son las cetariae, en las cuales se preparaba uno de los productos más relevantes en el flujo

$8 \mathrm{El}$ propio topónimo y la topografía actual, evidencian la naturaleza del terreno como zona de carácter lagunar. 
comercial antiguo; nos referimos concretamente a las salazones y productos derivados. Gracias a la cartografía histórica localizamos, aproximadamente el límite costero que pudo existir durante el s. XVII y sobre todo el s. XVIII ${ }^{9}$, fecha previa a las grandes modificaciones; permitiéndonos intuir una hipotética línea de costa antigua.

El siguiente paso ha sido geolocalizar algunas de estas factorías (Fig. 4) documentadas en Extramuros próximas al litoral interno de la Bahía (Expósito, 2006). En este sentido, podemos advertir que estas estructuras se localizaban mucho más cerca de la línea de costa de lo que podríamos concluir visualizando un plano actual. Por ello, la ubicación de dichas estructuras cobra mayor sentido, dado que este tipo de construcciones tendían a localizarse en zonas próximas al litoral, para facilitar el transporte del producto manufacturado y la materia prima por mar. Gracias a este ejercicio, detectamos que la proximidad sería aún mayor, favoreciendo el transporte interno por la Bahía y los trabajos de carga y descarga del producto.

\section{4.- CONCLUSIONES}

En primer lugar debemos destacar la gran utilidad, no solo de la cartografía como fuente de información, sino también el papel de los SIG en este tipo de estudios espaciales. Gracias a las herramientas como la georreferenciación o vectorización se pueden representar de forma gráfica todos los procesos evolutivos que hemos estudiado, permitiendo acometer análisis comparativos combinando información de diferentes cronologías.

Por otro lado, consideramos que a partir de los dos supuestos analizados en este trabajo, queda plenamente de manifiesto la funcionalidad de los planos y mapas del s. XVII, XVIII y XIX para estudiar paisajes antiguos, salvando el amplio marco cronológico que los separa. La cartografía histórica nos aporta valiosa información gráfica sobre la naturaleza morfológica de un enclave concreto; lo que facilita, en cierto modo, la idealización de la realidad paisajística que nos transmite sin necesidad de aplicar métodos invasivos, podemos establecer una línea evolutiva aproximada de las diferentes modificaciones que experimenta el litoral, combinándola con información literaria y arqueológica.

9 Recordemos que la cartografía histórica previa a las grandes transformaciones por antropización, se consideran un testimonio muy cercano a la realidad paisajística que pudo dominar en la Antigüedad

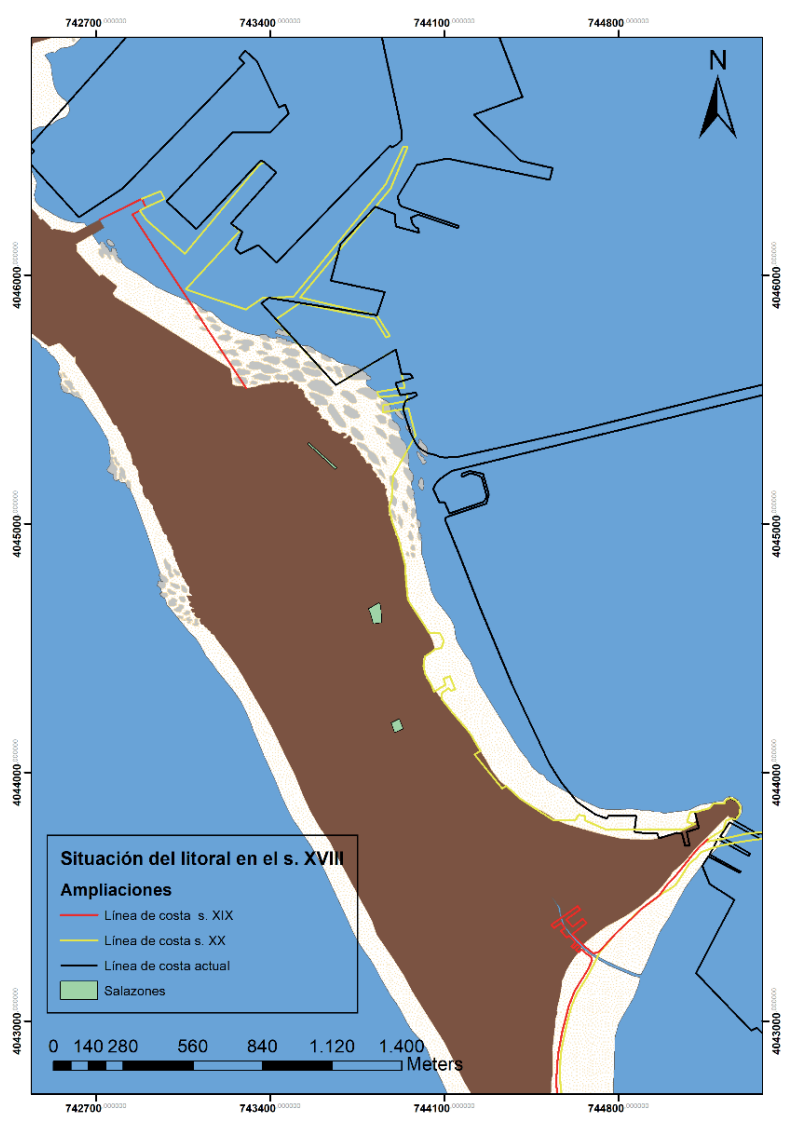

Figura 4.- Mapa de elaboración propia que representa la costa de Cádiz durante el s. XVIII (Museo Nacional. MN, E-52-40) en el cual se superpone las diferentes líneas de costa documentadas en los s. XIX (Instituto Geográfico Nacional. S1-35-N-34), s. XX (Real Observatorio de la Armada. ROA- 2930) y en la actualidad (Perfil costero PNOA_MA_OF_ETRS89_HU29_h50_1061. Centro Nacional de Información Geográfica). Junto a él los mapas originales que hemos utilizado para el análisis.

Y en tercer lugar, en relación a esta última disciplina, es importante destacar la relación entre la utilidad de la cartografía histórica, como fuente para conocer la evolución del litoral, con los datos arqueológicos que se ubican próximos a la costa interna de Cádiz. En este sentido, los datos cartográficos y los datos arqueológicos van de la mano hacia una misma dirección, contextualizar los yacimientos arqueológicos en su entorno natural. Como podemos advertir en la figura 4 las factorías de salazón se encontrarían mucho más próximas a la costa de lo que realmente se encuentran hoy en día, por causa de la acusada transformación del litoral. En este sentido podemos ver una clara orientación de estas factorías hacia la playa que dominaba gran parte del litoral interior del istmo. 
Además el vínculo con la arqueología, no solo estriba en la contextualización de yacimientos, como citamos anteriormente, sino también en la planificación de campañas arqueológicas y geoarqueológicas. Un claro ejemplo lo tenemos en el primer caso estudiado en el que la cartografía nos mostraba la naturaleza originaria del estuario del Guadalete, reflejando toda la zona en un estado avanzado de colmatación. De este modo, con una correcta georreferenciación y digitalización de los límites de estos espacios, puede ayudar a la futura programación de intervenciones en donde el objetivo sea, por ejemplo, estudiar cómo se produjo la colmatación del estuario o localizar la extensión real de dicho estuario.

\section{5.- BIBLIOGRAFÍA}

- Alonso, C., Benítez, D., Márquez, L. (2011), "Los documentos cartográficos como fuente de información para el conocimiento del Patrimonio cultural marítimo y fluvial", Revista $P H$, IAPH. $N^{a} 77$ (año $N^{a} 19$ ), 82-84.

- Alonso, C. A, Gracia, F. J y Rodríguez-Polo, S. (2014), "Modelo de evolución histórica de la flecha-barrera de Valdelagrana (Bahía de Cádiz)", XIII Reunión Nacional de Geomorfología, Cáceres. 584-587

- Alonso, C. A, Gracia, F. J y Rodríguez-Polo, S., Marín, C. (2015), "El registro de eventos energéticos marinos en la bahía de Cádiz durante épocas históricas", Cuaternario y Geomorfología, 29 (1-2). 95-117.

- Andrés, G. (2016), "Georreferenciación de planos antiguos mediante sistemas de información geográfica (SIG): una herramienta innovadora para la enseñanza de la historia urbana”, VIII Jornadas de Innovación Docente, Burgos.

-Arruda, A. M. (2005), "La navegación en el Atlántico. Las condiciones físicas y las evidencias arqueológicas”, Cuadernos de Arqueología Mediterránea, Vol. 5-6.23-30.

- Ash, A. (2007), The Maritime Cultural Landscape of Port Willunga, South Australia, Flinders university maritime archaeology monographs series Number 4, Flinders.

-Cobos, D. (1993), "La Bahía de Cádiz. Aproximaciones a un diagnóstico cartográfico de la evolución del paisaje”, Cuadernos de Geografía, 4.137-154

- Blanchemanche, Ph. (2000), "La plaine de lettes du XIIe au XIXe siècle. Dynamique naturelle et mise en valeur", Lattara 13.

- Cascón Katchadourian, J., Ruiz Rodríguez, A. A. y Alberich Pascual, J.(2017), "Usos y geolocalización en gestión documental cartográfica y fotográfica antiguas”. El profesional de la información, 2018, enero-febrero, v. 27.En línea: http: / / www.elprofesionaldelainformacion. com/contenidos/2018/ene/19.pdf (Consulta 15-IX-2018)

- Cerezo, F. (2013), "Paisajes culturales marítimos. Georreferenciación de planos históricos", I Congreso de Arqueología Náutica y Subacuática española. Ministerio de educación, cultura y deporte, Murcia, 469-475.

-(2016), Los puertos antiguos de Cartagena. Geoarqueología, arqueología portuaria, paisaje marítimo. Un estudio desde la Arqueología náutica, Tesis Doctoral, Murcia.

- Dávila, F. J y Camacho, E. (2012), “Georreferenciación de documentos cartográficos para la gestión de Archivos y Cartotecas. Propuesta Metodológica", Revista catalana de geografía, IV época, vol. XVII, $n^{\circ} 46$. V Ibercarto.

-Expósito Álvarez, J. A. (2006), “¿Dónde se encuentran las cetariae de Gades? Revisión arqueológica y estado de la cuestión sobre el emplazamiento de las factorías de salazón romanas de la ciudad de Cádiz", Actas del Congreso Internacional CETARIAE. Salsas y salazones de pescado en Occidente durante la Antigüedad, Cádiz, 367-385.

- Iglesias Rodríguez, J. J. (2017), "Las infraestructuras portuarias de la Bahía de Cádiz ante el reto del monopolio americano", Estudios de Historia Moderna, 39, n.2.185-192.

- López Amador, J. y Pérez Fernández, E. (2013), El Puerto Gaditano de Balbo, Cádiz.

- Martí, J. y Rodríguez, N. (2003), "Problemática y situación actual de la arqueología subacuática de la bahía de Cádiz”, Monte Buciero, 9, 397-416.

- Westerdahl, C (1980), "The Maritime Cultural Landscape: On the concept of the traditional zones of transport geography". En línea: https:// www.abc.se/ pa/publ/cult-land.htm (Consulta 15-I-2018)

- (1992), "The Maritime Cultural Landscape". The International Journal of Nautical Archaeology. 21 .1. 5-14. 
